\title{
The learning experience for endoscopic submucosal dissection in a non-academic western hospital: a single operator's untutored, prevalence-based approach
}

\author{
Georgios Mavrogenis ${ }^{a}$, Dimitrios Ntourakis ${ }^{b}$, Zhen Wang ${ }^{c}$, loannis Tsevgasa ${ }^{a}$ Dimitrios Zachariadis ${ }^{a}$, \\ Nikolaos Kokolas ${ }^{d}$, Loukas Kaklamanise, Fateh Bazerbachif \\ Mediterraneo Hospital, Athens, Greece; European University of Cyprus, Nicosia, Cyprus; Evidence-Based Practice \\ Center, Robert D. and Patricia E. Kern Center for the Science of Health Care Delivery, Mayo Clinic, Rochester, MN, \\ USA; Onassis Cardiac Surgery Center, Athens, Greece; CentraCare, St Cloud Hospital, Interventional Endoscopy \\ Program, St Cloud, MN, USA
}

\section{Abstract}

\section{Introduction}

Endoscopic submucosal dissection (ESD) has been established as a minimally invasive endoscopic treatment

\section{Conflict of Interest: None}

Correspondence to: Georgios Mavrogenis, Director of Hybrid Interventional Endoscopy, Department of Gastroenterology,

Mediterraneo Hospital, Ilias 12, 16675 Athens, Greece,

e-mail: mavrogenis@gmail.com

Received 23 January 2021; accepted 11 March 2021;

published online 2 July 2021

DOI: https://doi.org/10.20524/aog.2021.0649
Background Studies of learning experience in endoscopic submucosal dissection (ESD) commonly originate from the East. Little is known about the performance of ESD in low-volume western centers. Furthermore, it is unclear whether ESD can be self-taught without a tutored approach.

Methods We performed a retrospective analysis of consecutive ESDs, performed in an untutored prevalence-based fashion by a single operator at a private Greek hospital from 2016-2020. Out of 60 lesions, standard ESD was applied for 54 and enucleation for 6; 41 were mucosal and 19 submucosal; 3 esophageal, 24 gastric, one duodenal, 12 colonic, and 20 rectal.

Results Pathology revealed carcinoma $(n=14)$, neuroendocrine tumor $(n=7)$, precancerous lesion $(\mathrm{n}=27)$, or other submucosal tumors $(\mathrm{n}=12)$. The rates of en bloc and R0 resection were $98 \%$ and $91 \%$, respectively. The median resection speed was $<3 \mathrm{~cm}^{2} / \mathrm{h}$ for the first 20 cases, but improved progressively to $\geq 9 \mathrm{~cm}^{2} / \mathrm{h}$ after 40 cases. Two patients underwent laparoscopic surgery for colonic perforation, and one received a blood transfusion because of delayed bleeding (serious adverse event rate: $5 \%$ ). No deaths occurred. The median hospital stay was 1.3 days. Variables associated with improvement in ESD speed during the second period of the study were the application of countertraction and the experience acquired through other endosurgical techniques.

Conclusions ESD was safe and effective in a low-volume center, with an acceptable adverse events rate. At least 40 mixed cases were needed to achieve a high resection speed. Additive experience gained through other endosurgical procedures probably contributed to the improvement in performance.

Keywords Endoscopic submucosal dissection, third-space endoscopy, gastrointestinal cancer, endoscopy education, non-academic hospital

Ann Gastroenterol 2021; 34 (x): 1-9 for the curative resection of foregut and hindgut mucosal and submucosal pathology [1]. However, ESD remains a challenging procedure that usually requires extensive ex vivo and in vivo training, observation of several live cases and finally, hands-on tutored training in a high-volume center, or proctorship by an expert [2].

Though several animal workshops are available, hands-on training opportunities in humans remain limited, and ESD is not typically part of the advanced endoscopy curriculum in the West [3]. Moreover, training opportunities in the East are not broadly available, and the recent COVID-19 pandemic has hindered the scarce existing opportunities for such training.

Current evidence about the learning experience for ESD comes from high-volume referral hospitals [4-7]. 
These reports are potentially biased, as they emanate from skilled endoscopists, likely to be experienced in many other endoscopic techniques. When studies report the progress of junior endoscopists, they may also be heavily influenced by the involvement of an experienced tutor. A caseload of at least 20 gastric/rectal cases, with the first 10 cases supervised by a tutor, has been recommended before handling lesions in other locations, based on recent guidelines by the European Society of Gastrointestinal Endoscopy (ESGE) [2]. However, in real life, and with limited access to expert centers, the patient may need a proximal colon or esophageal ESD before the available operator has accomplished the ideal caseload of gastric-rectal ESDs, and without the luxury of an onsite instructor. This leaves surgery, with its known risks, as the only available option for the patient. Indeed, a real-life ESD practice outside of the confines or reach of a tertiary referral center will probably not follow the ideal complexity progression. In this environment, the operator has to address the technical difficulties on their own to build their repertoire and reputation, and provide patients with a safe and effective option. These realities may challenge the development of a successful, efficient and safe ESD program in low-volume non-academic centers. This study aims to evaluate the safety and efficacy of a self-taught, unsupervised ESD-program in a private, low-volume, and non-academic Greek hospital by a junior endoscopist, trained solely in the West.

\section{Patients and methods}

In a medium-sized (160 beds) non-academic private Greek hospital (Mediterraneo Hospital), a prospectively maintained database of all endosurgical procedures was queried from inception in March 2016 until December 2020. This database also included peroral endoscopic myotomy (POEM) and laparoscopic endoscopic cooperative surgery (LECS) for submucosal gastric tumors, in addition to ESD.

ESD was undertaken based on the indications published by the ESGE [1]. We also included patients with small submucosal lesions $(<3 \mathrm{~cm})$ that fulfilled one or more of the following criteria: 1) symptomatic lesion (e.g., obstruction, pain); 2) endoluminal lesion that appeared to emanate from the muscularis propria on endoscopic ultrasound (EUS); and 3) growing lesions of unknown origin when the EUS evaluation was inconclusive.

ESD was not offered in any of the following: suspicion of deep submucosal invasion; high-risk comorbidities with an

\footnotetext{
${ }^{a}$ Division of Hybrid Interventional Endoscopy, Department of Gastroenterology, Mediterraneo Hospital, Athens, Greece (Georgios Mavrogenis, Ioannis Tsevgas, Dimitrios Zachariadis); ${ }^{\mathrm{b}}$ Department of Surgery, School of Medicine, European University of Cyprus, Nicosia,

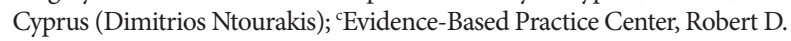
and Patricia E. Kern Center for the Science of Health Care Delivery, Mayo Clinic, Rochester, MN, USA (Zhen Wang); ' dDepartment of Anesthesiology, Mediterraneo Hospital, Athens, Greece (Nikolaos Kokolas); ${ }^{\mathrm{e} D e p a r t m e n t}$ of Pathology, Onassis Cardiac Surgery Center, Athens, Greece (Loukas Kaklamanis); ${ }^{\mathrm{f} C e n t r a C a r e, ~ S t ~ C l o u d ~ H o s p i t a l, ~ I n t e r v e n t i o n a l ~ E n d o s c o p y ~}$ Program, St Cloud, MN, USA (Fateh Bazerbachi)
}

expected short survival time or comorbidities prohibitive of surgery, if a complication was to occur; coagulopathy; or pregnancy. Submucosal lesions with an exophytic extraluminal component were treated by LECS, as previously reported [8-10]. The risks and benefits of ESD, along with alternative options, were discussed with the patients, and all patients gave consent to proceed with ESD.

\section{Operator}

The single operator (GM) was fellowship-trained in endoscopic mucosal resection, EUS and endoscopic retrograde cholangiopancreatography (ERCP), and initiated the ESD program at Mediterraneo Hospital, one year after completing a gastroenterological fellowship in Europe.

Prior training included: (i) intermittent observation of ESD and POEM procedures during a 4 -year residency program in 2 high-volume European referral centers (first instructor: Dr. Dimitri Coumaros, Nouvel Hôpital Civil, Strasbourg, France, and second instructor Prof. Pierre Deprez, Cliniques Universitaires Saint Luc, Brussels, Belgium); (ii) ex vivo gastric ESDs in pigs $(n=20)$; (iii) in vivo gastric ESDs in pigs $(n=6)$; and (iv) indirect exposure to Japanese ESD experience through online resources.

Furthermore, during the period of ESD cases \#23-\#60, the operator also performed 13 POEMs, 2 gastric LECS, and participated as an instructor in 4 annual ESD hands-on animal workshops organized by the European Association for Gastroenterology, Endoscopy and Nutrition and the Hellenic Society of Gastroenterology [11].

\section{ESD procedure}

All procedures were carried out under general anesthesia, apart from rectal cases, performed under propofol sedation. Gastric and rectal cases were performed in the endoscopy unit, whereas esophageal and colon cases were performed in the operating theater.

On the day of the procedure, the patients received $4.5 \mathrm{~g}$ of piperacillin/tazobactam. Patients with upper gastrointestinal lesions received a daily dose of $40 \mathrm{mg}$ of esomeprazole the week before the procedure.

Procedures were performed with either a GIF 1TH-190, PCF-190 or CF-185 (Olympus, Tokyo, Japan). A disposable distal attachment (D-201-12704/804, Olympus), $\mathrm{CO}_{2}$ insufflation and a water pump with $0.9 \%$ saline were used. A tapered tip hood was used as needed for tunneling (STHood, Fujifilm, Japan, Tokyo).

A cutting-tip knife was used in all cases. A ceramic tip knife was also applied as needed for areas with perpendicular access. The submucosa was injected with a mixture of hydroxyethyl starch, indigo carmine and epinephrine: $1 \mathrm{~mL}$ of indigo carmine $(40 \mathrm{mg} / 5 \mathrm{~mL}), 1 \mathrm{~mL}$ of epinephrine $(1 \mathrm{mg} / \mathrm{mL})$ in $500 \mathrm{~mL}$ of hydroxyethyl starch. A coagrasper (Olympus) was used for hemostasis, as needed. 
Two different electrosurgical units were used, a VIO200D or VIO3 (ERBE Elektromedizin GmbH, Tübingen, Germany) with the following settings: Endocut Q, E2-3 or Drycut, E3 for mucosal incision; Swift Coag E3, Forced Coag E3 or Spray Coag, E3 for submucosal dissection; Soft Coag E4 for hemostasis.

In terms of dissection strategy, circumferential incision and then standard dissection was applied for gastric and esophageal lesions, while tunneling techniques [12,13] were mainly applied for duodenal and colorectal lesions. Additional techniques included the saline immersion technique [14] and countertraction, such as the clip-and-snare [15], the clipand-line and the clip-and-band techniques $[14,16]$. However, from case \#40 we systematically applied standard dynamic or multifocal clip-and-band countertraction [16] to increase the dissection speed and diminish the perforation risk.

Submucosal tumors were resected by the standard ESD technique or by tunneling and enucleation [17], depending on the morphology and relation to the muscle layer. At the end of the procedure, all visible vessels were meticulously coagulated. Clips were placed in areas with large vessels or muscular injury when deemed necessary. Complete closure was preferred, particularly for colonic lesions and in patients needing antithrombotics/antiaggregants.

\section{Definition of adverse events (AEs)}

In general, post-procedural bleeding was defined as significant blood loss and hemoglobin drop $>2 \mathrm{~g} / \mathrm{dL}$ or as overt bleeding (e.g. hematemesis, melena) requiring hospitalization or endoscopic treatment. Intraprocedural perforation was defined as a penetrating muscular layer injury with visible serosa or fat. The ASGE lexicon for endoscopic AEs was applied to determine the severity of the event [18].

\section{Histopathological assessment}

Resected specimens were pinned and immersed in $10 \%$ formalin solution and sectioned serially at 2-mm intervals. Attention was paid to the horizontal and vertical margins, and when malignancy was present, to the presence of lymphovascular infiltration, the grading of tumor budding, and the depth of submucosal invasion.

\section{Statistical analysis}

ESD procedural duration was defined as the time between submucosal injection and specimen retrieval. Procedural speed $\left(\mathrm{cm}^{2} / \mathrm{h}\right)$ was calculated on the assumption that every lesion had approximately a circular shape, and the $A=\pi r^{2}$ formula was used. The rates of en bloc resection, R0 resection and the average resection speed were calculated, each for sequential blocks of 10 cases. Statistical comparisons were also performed between the first (cases 1-30) and second (cases 31-60) period. Submucosal lesions resected by enucleation were counted in each block but not included in the ESD speed analysis. Variables were tested with the Kruskal-Wallis test, McNemar's test, chi-square test, or Fisher's test. Statistical analysis was performed using IBM SPSS Statistics for Mac, version 25.0 (IBM Corp. Armonk, NY).

\section{Case series risk of bias}

We relied on a widely-used tool to provide a quality assessment of the risk of bias in our reported case series [19]. This tool has been applied in previous studies, with consistency among reviewers [20-25]. All patients represented the whole experience of the operator during the study period, and case inclusion was not biased (all qualified patients were included without omission). The exposure (ESD) was adequately described and ascertained for all cases. The outcomes (learning efficiency outcomes and safety) were described and ascertained in all cases. Factors that influenced the desired outcomes were considered. Follow up was adequate for the assessment of the outcome.

\section{Results}

\section{Patients and lesion characteristics}

Between March 2016 and December 2020, 60 ESDs were performed in 57 patients for 41 mucosal lesions and 19 submucosal lesions (Table 1). Tunneling and enucleation was used to resect 6 submucosal lesions, while the rest underwent standard ESD. Between 2018 and 2020 the operator performed 13 peroral endoscopic myotomies (11 for achalasia, 2 for Zenker's diverticulum) and 2 LECS.

Of the lesions, 3 were esophageal, 24 gastric, 1 duodenal, 12 colonic ( 5 cecum, 2 ascending colon, 3 transverse colon, 2 sigmoid colon) and 20 rectal (Figs. 1-3). For the 41 mucosal lesions, the pathology was 24 low-grade dysplasia, 11 highgrade dysplasia/in situ cancer, 3 cancer with submucosal invasion, and 3 gastric hyperplastic polyps. For the submucosal lesions, pathology showed 7 neuroendocrine tumors, 7 lipomas, 2 inflammatory fibroid polyps, and 1 leiomyoma.

\section{Learning experience analysis}

The en bloc resection was achieved in 59/60 (98\%) of lesions. One lesion in the right colon with low-grade dysplasia (case \#20) was removed piecemeal, after inadvertent perforation at the beginning of dissection.

R0 resection was achieved in 49/54 (91\%) of ESD cases. The 5 cases that did not achieve R0 resection included case $\# 20$, as previously mentioned; 3 rectal cases (\#7, \#44, and \#52) removed en bloc, but histology showed deep submucosal invasion (early $\mathrm{T} 2, \mathrm{sm} 2$, and $\mathrm{sm} 2$, respectively) with positive vertical margins and negative horizontal margins; and 1 gastric fibroid inflammatory polyp (\#3), removed with positive vertical margins. 
Table 1 Patients' demographics, lesion characteristics, and procedure parameters

\begin{tabular}{|c|c|c|c|c|}
\hline \multirow[t]{2}{*}{ Variable } & \multirow[t]{2}{*}{ Total } & \multicolumn{2}{|c|}{ Learning phase } & \multirow[t]{2}{*}{ P-value } \\
\hline & & First phase & Second phase & \\
\hline Lesions (n) & 60 & 30 & 30 & \\
\hline Standard ESD (n) & 54 & 27 & 27 & \\
\hline ESD with enucleation (n) & 6 & 3 & 3 & \\
\hline Tumor size $(\mathrm{cm})$ & $3.15(0.6-9.8)$ & $3(0.7-9.8)$ & $4(0.6-9)$ & 0.052 \\
\hline Surface $\left(\mathrm{cm}^{2}\right)$ & $7.8(0.28-78.5)$ & $7.07(0.38-78.5)$ & $12.56(0.28-48.16)$ & 0.049 \\
\hline Duration (min) & $120(12-600)$ & $135(18-600)$ & $105(12-270)$ & 0.07 \\
\hline Dissection Speed $\left(\mathrm{cm}^{2} / \mathrm{h}\right)^{*}$ & $3.8(0.4-25.6)$ & $3.1(0.4-19.60)$ & $8.6(1.6-25.6)$ & $<0.001$ \\
\hline \multicolumn{5}{|l|}{ Location } \\
\hline Stomach & & 13 & 11 & \\
\hline Non-antral & 24 & 8 & 4 & 0.14 \\
\hline Rectum & 12 & 8 & 12 & \\
\hline With anal involvement & 20 & 2 & 7 & 0.19 \\
\hline Colon & 12 & 6 & 6 & \\
\hline Cecum & 5 & 3 & 2 & \\
\hline Ascending & 2 & 1 & 1 & \\
\hline Transverse & 3 & 1 & 2 & \\
\hline Descending & 0 & 0 & 0 & \\
\hline Sigmoid & 2 & 1 & 1 & \\
\hline Duodenum & 1 & 1 & 0 & \\
\hline Esophagus & 3 & 2 & 1 & \\
\hline Mucosal lesion & 41 & 19 & 22 & \\
\hline Submucosal lesion & 19 & 11 & 8 & \\
\hline Fibrosis & $8(13 \%)$ & $6(20 \%)$ & $2(6 \%)$ & 0.25 \\
\hline Countertraction & $17(28 \%)$ & $5(17 \%)$ & $12(40 \%)$ & 0.039 \\
\hline Perforation & $6(10 \%)$ & $4(13 \%)$ & $2(6 \%)$ & 0.67 \\
\hline Need for surgery & & $1(3 \%)$ & $1(3 \%)$ & \\
\hline Bleeding & $3(5 \%)$ & 0 & $3(10 \%)$ & \\
\hline Need for transfusion & $1(3 \%)$ & 0 & $1(3 \%)$ & \\
\hline \multicolumn{5}{|l|}{ Histology } \\
\hline Hyperplastic & 3 & 1 & 2 & \\
\hline Low-grade dysplasia & 24 & 11 & 13 & \\
\hline High-grade dysplasia/in situ carcinoma & 11 & 6 & 5 & \\
\hline Submucosal invasion $>$ sm 1 & 3 & 1 & 2 & \\
\hline En bloc resection & $59(98 \%)$ & $29(97 \%)$ & $30(100 \%)$ & \\
\hline R0 Resection* & 49 (91\%) & $24(88 \%)$ & $25(92 \%)$ & \\
\hline + horizontal margins & $1(2 \%)$ & $1(4 \%)$ & 0 & \\
\hline + vertical margins & $4(7 \%)$ & $2(7 \%)$ & $2(7 \%)$ & \\
\hline Hospital stay & $1(0-3)$ & $1(0-3)$ & $1(0-3)$ & \\
\hline Other ESD-related procedures & 15 & $\begin{array}{c}4 \\
(4 \mathrm{POEM})\end{array}$ & $\begin{array}{c}11 \\
\text { (7 POEM, } 2 \text { Z-POEM, } 2 \text { LECS) }\end{array}$ & 0.037 \\
\hline
\end{tabular}

${ }^{*}$ Submucosal tumors $(\mathrm{n}=6)$ resected by enucleation not included

ESD, endoscopic submucosal dissection; POEM, peroral endoscopic myotomy; LECS, laparoscopic endoscopic cooperative surgery; Z-POEM, Zenker's POEM

The median lesion diameter was 3.15 (interquartile range [IQR] 0.6-9.8) cm. Median surface area was 7.8 (IQR 0.28$78.50) \mathrm{cm}^{2}$. Median ESD duration was 120 (IQR 12-600) $\mathrm{min}$.

Complete closure of the defect was performed with clips in $23(38 \%)$ cases, targeted clips without full closure in 19 (32\%) cases, while no clips were used in 18 (30\%) cases.
The median ESD procedure speed in the first 10 cases was 2.7 (IQR $0.4-9.4$ ) $\mathrm{cm}^{2} / \mathrm{h}$ and had not increased by the second 10 -case block. Thereafter, we noted a progressive increase in the following periods with median values of 4.9 (IQR 1.7-19.6) $\mathrm{cm}^{2} / \mathrm{h}$ in the third 10 cases block, 7.1 (IQR $1.6-16.7$ ) $\mathrm{cm}^{2} / \mathrm{h}$ in the fourth block, 9 (IQR 3.4-25.6) $\mathrm{cm}^{2} / \mathrm{h}$ in the fifth block, and 


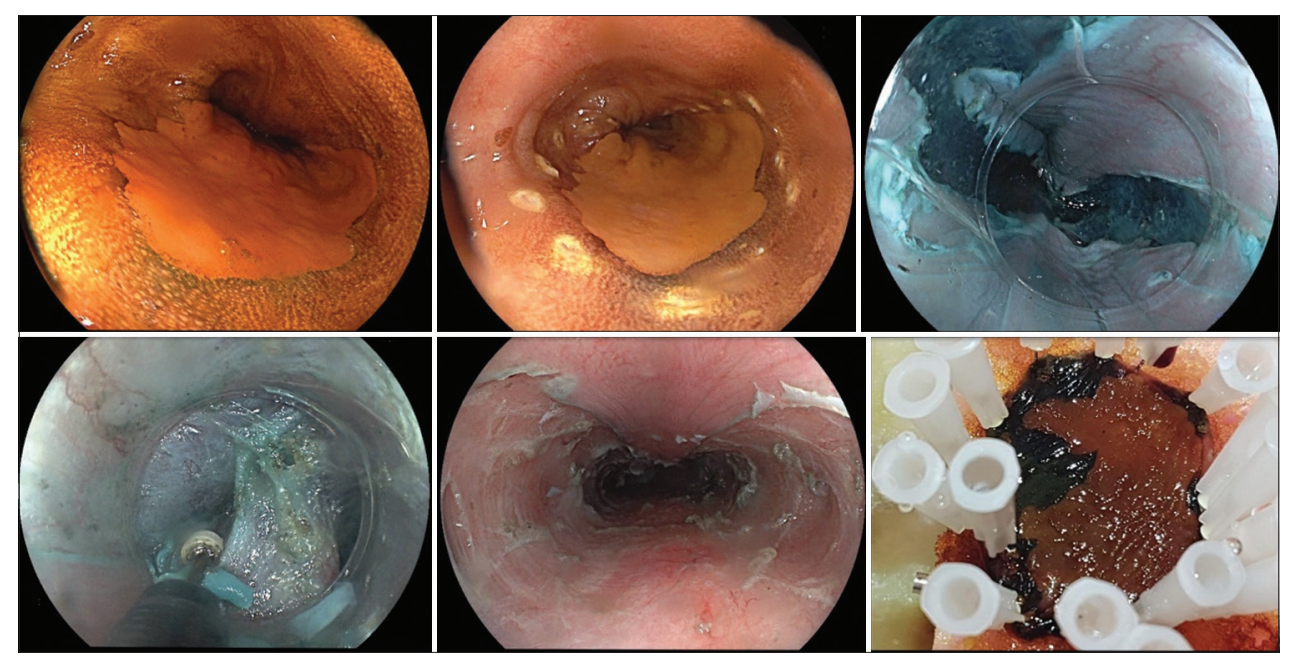

Figure 1 Endoscopic submucosal dissection for high-grade dysplasia of the proximal esophagus. R0 resection

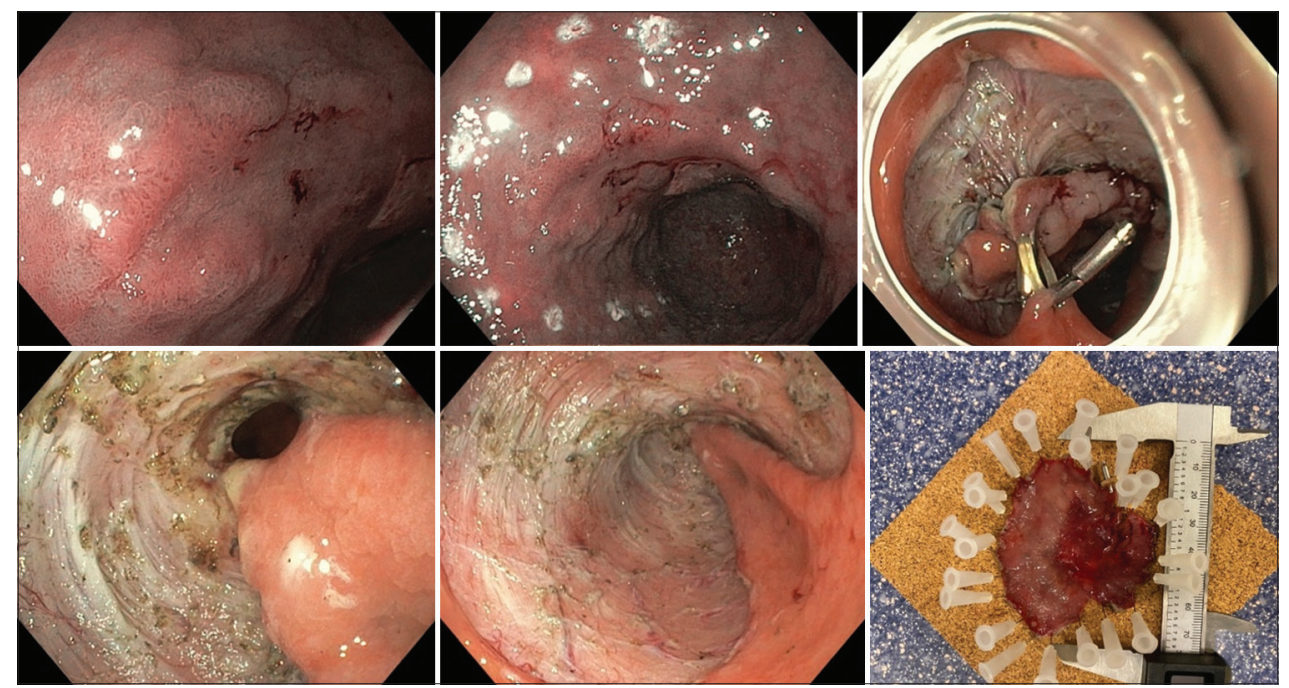

Figure 2 Clip and band endoscopic submucosal dissection of the whole antrum for intestinal metaplasia with multifocal low- and high-grade dysplasia. R0 resection

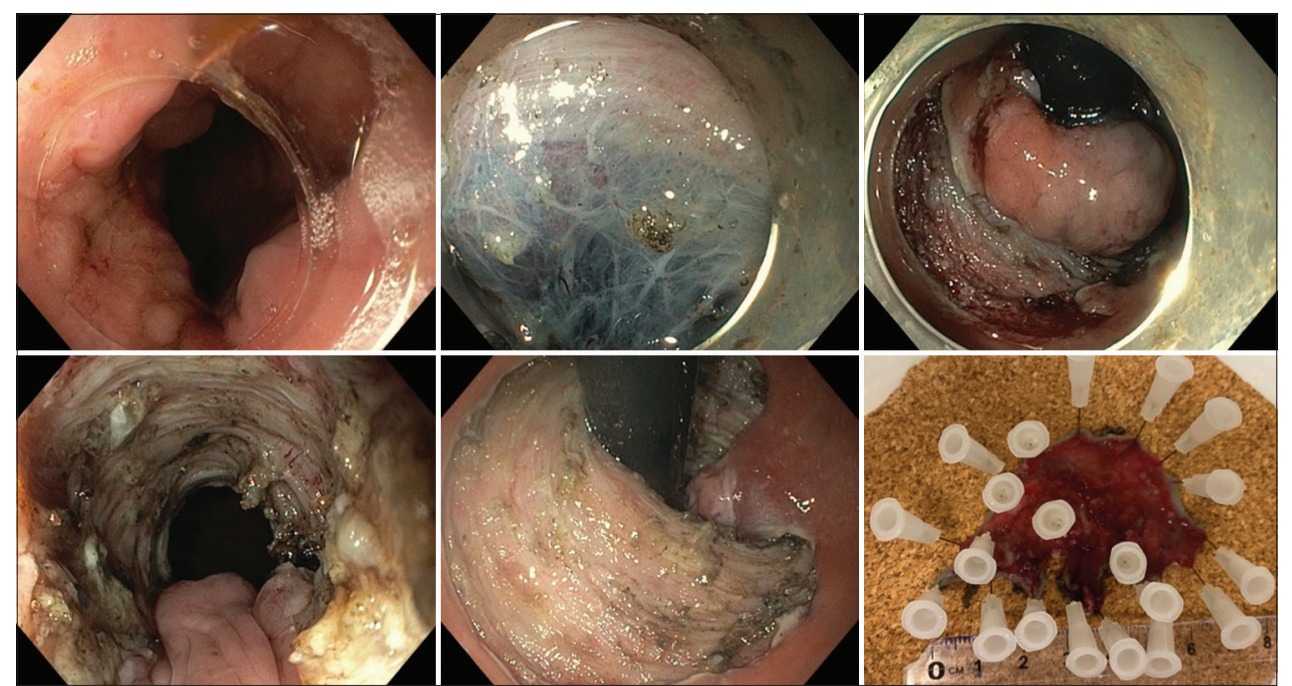

Figure 3 Endoscopic submucosal dissection for a tubulovillous anorectal adenoma with multifocal high-grade dysplasia. R0 resection 
9.4 (IQR 3-24.5) $\mathrm{cm}^{2} / \mathrm{h}$ in the sixth block (Fig. 4). A statistically significant increase in the median speed was noted between the first block and the last 2 blocks $(\mathrm{P}=0.023$ and $\mathrm{P}=0.031$, respectively), as well as between the second block and the last 2 blocks $(\mathrm{P}=0.012$ and $\mathrm{P}=0.017$, respectively). Thus, the benchmark of a high resection speed $\left(\geq 9 \mathrm{~cm}^{2} / \mathrm{h}\right)$ was reached in the last 2 blocks, after 40 cases had been performed.

The median resection speed per organ was as follows: 8.7 (IQR 1.6-25.6) $\mathrm{cm}^{2} / \mathrm{h}$ in the rectum, 3.9 (IQR 0.4-24.5) $\mathrm{cm}^{2} / \mathrm{h}$ in the stomach, $3.1 \mathrm{~cm}^{2} / \mathrm{h}$ (IQR 1.20-16.7) in the colon, and 3.2 (IQR $1.7-3.7) \mathrm{cm}^{2} / \mathrm{h}$ in the esophagus. However, these differences were not statistically significant $(\mathrm{P}=0.22)$ (Fig. 5).

\section{Comparison of the first and second half of the study}

Notably, the improvements in dissection speed were present despite the increase in lesion size between the first and second periods of the series: only $26 \%$ of lesions were $\geq 4 \mathrm{~cm}$ in the first period compared with $61 \%$ in the second period $(\mathrm{P}=0.018)$. The median speed increased significantly from 3.1 (0.4-19.6) $\mathrm{cm}^{2} / \mathrm{h}$ in the first half, to $8.6(1.6-25.6) \mathrm{cm}^{2} / \mathrm{h}$ in the second period $(\mathrm{P}=0.001)$. We examined several variables between the first and second periods to detect factors associated with the higher resection speed. Although the lesions were statistically significantly larger in the second period, the proportion of gastric/colon cases, the anatomic location of the lesions and the presence of fibrosis did not differ significantly. However, the

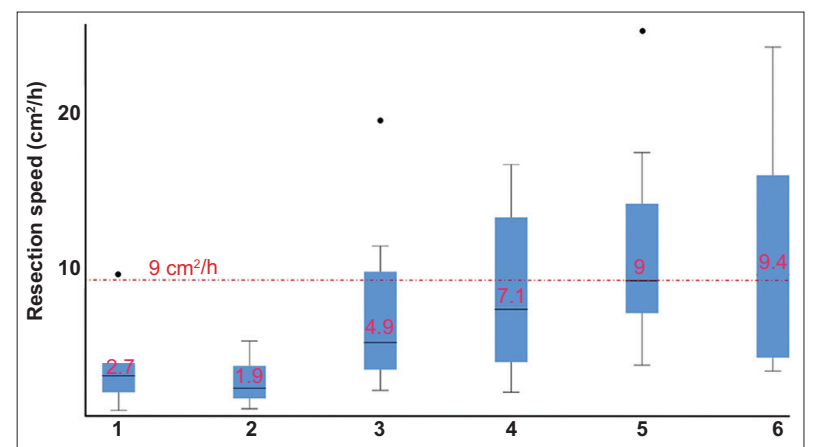

Figure 4 The trend of median resection speed over sequential blocks of 10 cases

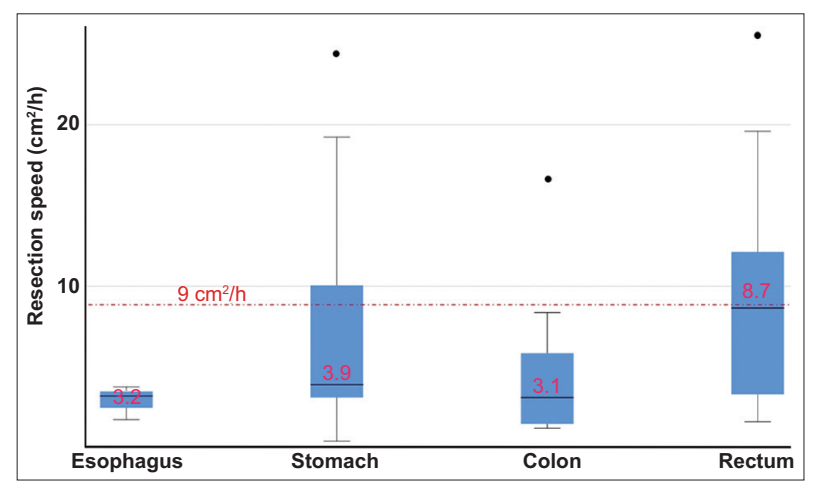

Figure 5 Median resection speed for each location application of countertraction was significantly more frequent in the second period ( $40 \%$ vs. $17 \%, \mathrm{P}=0.039$ ), and the operator performed more endosurgical procedures (POEM/LECS) in the second period ( 11 vs. $4, \mathrm{P}=0.04$ ).

\section{Analysis of AEs}

\section{Perforations}

We encountered 6 intraprocedural perforations; 4 were treated endoscopically, and 2 required surgery.

- Case \#15: A 5-mm perforation occurred during dissection of a $2-\mathrm{cm}$ gastric neuroendocrine tumor (NET) located in the proximal body. Endoscopic clips were placed, without interfering with further dissection. The patient was hospitalized for 2 days (mild AE).

- Case \#19: A 10-mm perforation in the sigmoid colon for a depressed non-granular lesion involving $2 / 3$ of the lumen located in a stenotic segment with severe fibrosis secondary to radiation. Despite clipping, the patient developed peritonitis and underwent laparoscopy $24 \mathrm{~h}$ after ESD. Surgical lavage of pus and placement of a drain was performed. The drain was removed after $24 \mathrm{~h}$. The patient was hospitalized for 3 days and had a fast and uneventful recovery (severe $\mathrm{AE}$ ).

- Case \#20: A 5-mm perforation in the ascending colon for a $3-\mathrm{cm}$ adenoma with low-grade dysplasia and postbiopsy fibrosis. The patient was managed conservatively with endoscopic clips, antibiotics and bowel rest and was hospitalized for 3 days (mild AE).

- Case \#28: A 5-mm perforation occurred during deep submucosal dissection of a gastric NET of the proximal body. The perforation was immediately clipped. The patient was discharged after 2 days (mild AE).

- Case \#32: A 5-mm perforation occurred in the transverse colon for a flat lesion expanding over a fold. Despite clipping, the patient developed peritonitis and underwent laparoscopy. Surgical lavage of pus and placement of a drain was performed for $24 \mathrm{~h}$. The patient was discharged on postoperative day 3 after drain removal and had a fast and uneventful recovery (severe AE).

- Case \#57: A 5-mm perforation occurred in the sigmoid colon during dissection of a 4-cm laterally-spreading mixed type granular adenoma. Clips were placed, and the patient was discharged after 3 days of hospitalization (mild AE).

\section{Bleedings}

Three patients presented with delayed bleeding.

- Case \#34: Minor rectal bleeding occurred $24 \mathrm{~h}$ after ESD of a 6-cm mid-rectum lesion. Hemoglobin level was stable. The bleeding was managed with repeat endoscopy and additional coagulation of the post-resection ulcer. The patient was discharged on postoperative day 1 (moderate AE). 
- Case \#38: Patient with atrial fibrillation and chronic anticoagulation underwent ESD for a $3.5-\mathrm{cm}$ rectal lesion with extension into the anal canal. Rivaroxaban was held 3 days before ESD, but the patient resumed therapeutic subcutaneous heparin as a bridge immediately after the procedure for 7 days. Severe rectal bleeding occurred 10 days after the ESD and hypotension ensued. The patient was hospitalized for 3 days, received 2 units of blood transfusions, and endoscopic clipping achieved hemostasis (severe AE).

- Case \#54: Resection of a 9-cm anorectal lesion. On postoperative day 3 the patient experienced minor bleeding, without intervention, and the hemoglobin level remained stable throughout (mild AE).

No other AEs were experienced. Specifically, patients did not experience post-resection abdominal pain or fever, and no deaths occurred.

\section{Risk of bias}

Our case series showed a low risk of bias in totality in all domains of quality assessment.

\section{Discussion}

ESD has become a standard treatment for early gastrointestinal neoplasia and complex colorectal lesions with high suspicion of limited submucosal invasion [1]. Despite the dissemination of ESD in the West, it is typically limited to high-volume academic centers and offered by a small number of experts in each country. However, several non-academic hospitals have recently introduced this procedure, with operators sharing their experience as case reports/series [12-17], surveys [26], and video uploads over the internet. Nonetheless, data regarding the quality and learning experience in these settings are lacking. Current literature suggests that ESD trainees should first practice in ex vivo and in vivo animal models, observe several cases performed by experts and then perform at least 20-50 gastric cases before embarking on ESD in other locations under the supervision of experienced proctors. However, in the West, early gastric neoplasia is less common than in the East, and detection is more challenging because of the lack of appropriate training and the absence of screening programs. Consequently, western endoscopists have limited opportunities to perform gastric ESD, postulated to be the easier initial step, and deemed safer than colorectal ESD.

However, recent data showed that endoscopists with limited exposure to gastric ESD ( $<5$ cases) might have acceptable initial en bloc rates, $\mathrm{R} 0$ resection rates and perforation rates: $88.3 \%, 75 \%$ and $10 \%$, respectively [27]. In addition, other reports show the efficacy of animal model training and self-learning software in the improvement of colorectal ESD skills [28]. These results may suggest that extensive gastric ESD experience is not a prerequisite for starting colorectal ESD.
In our single-operator retrospective experience, we show that ESD can be practiced safely and effectively by a junior endoscopist with broad training in interventional endoscopy during a gastroenterology fellowship, and without specific tutoring for a variety of lesions in a prevalence-based setting. The en bloc resection rate was $98 \%$ and remained stable over the entire study. Only one right colonic lesion was removed in a piecemeal fashion because of early perforation, which impeded further dissection progression. Subsequent similar lesions were resected en bloc, and success was aided by theimplementationofadditionaltechniques,suchasthepocketcreation method [12], the underwater saline immersion technique [14], and clip-and-band countertraction [14,17].

The $\mathrm{R} 0$ resection rate of $91 \%$ for the corresponding caseload is equal to or higher than that of previously published series from academic centers [4-7,29-32]. R0 was not achieved in 5 cases. Of these, 3 rectal specimens showed positive vertical margins due to deep submucosal invasion ( $\mathrm{sm} 2$ in 2 lesions and T2 in 1 lesion): these 3 patients were referred for radiotherapy because they refused surgery; 1 presented with local recurrence 3 months later but eventually died from a cardiac event 6 months later, and 2 patients had no evidence of recurrence at 1 -year post-resection.

One of the early quality goals in ESD implementation is to limit the rate of serious AEs to $<10 \%$ [29]. In our series, we encountered 3 serious AEs and one moderate AE, while the remaining 5 AEs were mild, according to the ASGE lexicon [18].

Most perforations occurred in the colon, of which 2 needed laparoscopic drainage for $48 \mathrm{~h}$. Importantly, no patient needed major salvage surgery (laparotomy, colectomy or colostomy). Of 6 perforations, 4 occurred in the first period compared with 2 in the second period. The perforation rate was lower in the second phase, probably because of improvement in the dissection dexterity and the introduction of tunneling, countertraction and underwater saline dissection. Therefore, more challenging ESD cases (such as lesions of the proximal stomach and colon) should be probably taken in charge in the later phase of the learning curve, once the trainee has successfully completed at least 20-30 antral or rectal cases where the risk of perforation is lower.

Among those patients with bleeding, 2 needed endoscopic hemostasis, but only 1 patient needed a blood transfusion, and this was in the setting of anticoagulation resumption within 2 weeks after the procedure.

There are several limitations to our current study that should be acknowledged. First, it is based on a retrospective evaluation, albeit of a prospectively maintained database. Second, it is limited by the small number of patients, although this caseload probably reflects the real-world referral pattern in a prevalence-based low-volume center. For example, a recent experience from a large tertiary referral center (in New York state, with a population of 19 million) analyzed 540 cases over a decade, with likely referrals from neighboring states and nationally [30]. In Greece, the population is 10 million, and ESD is not a widely performed procedure. Moreover, the current study period of 4 years is relatively shorter than other reported publications [30]. Third, the current analysis is based on the experience of a single operator, who underwent dedicated 
training in additional therapeutic endoscopy techniques (e.g., ERCP, POEM), and therefore may not be generalized to those only trained in basic endoscopy.

Despite these shortcomings, our results are provocative and relevant, as they highlight a few critical points. First, this is the only published analysis from Greece and one of the few emanating from a non-academic, low-volume private setting, showing a real-life prevalence-based experience. Nonetheless, good outcomes are demonstrated, from both efficacy and safety standpoints, despite the lack of ESD-specific tutoring (whether in an ESD center or by inviting onsite proctors), and despite the low caseload over the study period.

These results may suggest that self-education in ESD, including practice with animal models, workshop attendance, and self-study with online resources, may allow the development of an ESD program in low-volume centers, provided ample time is allocated for these cases and multidisciplinary support from the hospital and surgical staff is available. In addition, patients should be closely followed-up and their data collected meticulously for quality control.

In conclusion, we demonstrated the safety and feasibility of launching an ESD program in a low-volume hospital, without ESD-specific tutoring, by a junior endoscopist who had

\section{Summary Box}

\section{What is already known:}

- Endoscopic submucosal dissection (ESD) is a sophisticated technique that requires mastery for safe and effective performance

- Studies evaluating the learning experience of ESD originate from high-volume tertiary centers with onsite tutoring and carefully selected lesions of progressive difficulty

- Performance of $\geq 20$ cases of antral or gastric ESD is recommended before attempting ESD in other locations

\section{What the new findings are:}

- After appropriate self-developed training, ESD can be safely performed in a low-volume setting, without tutoring in a prevalence-based fashion, and with an acceptable adverse event profile by an endoscopist who has received structured training in ESD and other advanced techniques

- Dissection speed increased significantly after 20 mixed cases and reached the threshold of $9 \mathrm{~cm}^{2} / \mathrm{h}$ after 40 cases

- Variables associated with higher resection speed included the use of countertraction and the practice of other third-space endoscopy procedures, such as peroral endoscopic myotomy and laparoscopic endoscopic cooperative surgery received structured training in various advanced techniques (e.g., ERCP, POEM). All benchmarks of quality were met based on the current recommendations ( $>90 \%$ en bloc resection rate, $>80 \%$ R0 resection rate, resection speeds $>9 \mathrm{~cm}^{2} / \mathrm{h}$, and a serious AE rate of $<10 \%$ ) after a caseload of 40 cases. We further illustrate the positive impact of assisting techniques, such as countertraction, and the value of practicing other endosurgical procedures to improve the trajectory of ESD mastery.

\section{Acknowledgment}

The authors would like to thank the nurses of our endoscopy unit for their invaluable support: Mrs S. Fragidi, Mr A. Kostaras, Mrs I. Manoli, Mrs S. Kontogianni, Mrs D. Barla, Mrs K. Evagelou.

\section{References}

1. Pimentel-Nunes P, Dinis-Ribeiro M, Ponchon T, et al. Endoscopic submucosal dissection: European Society of Gastrointestinal Endoscopy (ESGE) Guideline. Endoscopy 2015;47:829-854.

2. Pimentel-Nunes P, Pioche M, Albéniz E, et al. Curriculum for endoscopic submucosal dissection training in Europe: European Society of Gastrointestinal Endoscopy (ESGE) Position Statement. Endoscopy 2019;51:980-992.

3. McCarty TR, Aihara H. Current state of education and training for endoscopic submucosal dissection: Translating strategy and success to the USA. Dig Endosc 2020;32:851-860.

4. Jeon HH, Lee HS, Youn YH, Park JJ, Park H. Learning curve analysis of colorectal endoscopic submucosal dissection (ESD) for laterally spreading tumors by endoscopists experienced in gastric ESD. Surg Endosc 2016;30:2422-2430.

5. Hong KH, Shin SJ, Kim JH. Learning curve for endoscopic submucosal dissection of gastric neoplasms. Eur J Gastroenterol Hepatol 2014;26:949-954.

6. Hotta K, Oyama T, Shinohara T, et al. Learning curve for endoscopic submucosal dissection of large colorectal tumors. Dig Endosc 2010;22:302-306.

7. Boda K, Oka S, Tanaka S, et al. Real-world learning curve analysis of colorectal endoscopic submucosal dissection: a large multicenter study. Surg Endosc 2020;34:3344-3351.

8. Ntourakis D, Michalinos A, Schizas D. Hybrid laparoscopic and endoscopic partial gastrectomy for ulcerated GIST: surgical technique with video. World J Surg 2020;44:202-206.

9. Ntourakis D, Mavrogenis G. Cooperative laparoscopic endoscopic and hybrid laparoscopic surgery for upper gastrointestinal tumors: Current status. World J Gastroenterol 2015;21:12482-12497.

10. Mavrogenis G, Mitropapas G, Kyriakidou V, Bazerbachi F. Laparoscopic endoscopic cooperative surgery for a gastric glomus tumor previously diagnosed by endoscopic ultrasound fine-needle biopsy. Ann Gastroenterol 2020;33:219.

11. Kasapidis P, Bassioukas S, Mavrogenis G, et al. Experimental gastric endoscopic submucosal dissection: training in a porcine model. Ann Gastroenterol 2017;30:446-449.

12. Mavrogenis G, Ntourakis D, Tsevgas I, Kaklamanis L, Zachariadis D. Pocket endoscopic submucosal dissection with countertraction and partial full-thickness excision as salvage therapy for advanced colonic adenoma with severe fibrosis. VideoGIE 2018;3:211-212.

13. Mavrogenis G, Tsevgas I, Kasapidis P, Zachariadis D. Single 
tunneling technique for the resection of lateral spreading adenomas. Ann Gastroenterol 2017;30:580.

14. Mavrogenis G, Mavrogenis I, Anastasiadis S, Bazerbachi F. Underwater endoscopic submucosal dissection in saline solution with rubber-band countertraction for a cecal polyp extending into a diverticulum. Ann Gastroenterol 2019;32:527.

15. Mavrogenis G, Georgousis N, Ntourakis D, Mavrogiorgis A. Clip and snare countertraction technique for rectal submucosal dissection. Ann Gastroenterol 2017;30:129.

16. Mavrogenis G, Bazerbachi F, Tsevgas I, Zachariadis D. Dynamic and multifocal clip and band countertraction for endoscopic submucosal dissection. VideoGIE 2020;5:451-454.

17. Mavrogenis G, Ntourakis D, Tsevgas I, Zachariadis D. Submucosal pocket-assisted enucleation of gastric submucosal lesions. Ann Gastroenterol 2017;30:466.

18. Cotton PB, Eisen GM, Aabakken L, et al. A lexicon for endoscopic adverse events: report of an ASGE workshop. Gastrointest Endosc 2010;71:446-454.

19. Murad MH, Sultan S, Haffar S, Bazerbachi F. Methodological quality and synthesis of case series and case reports. BMJ Evid Based Med 2018;23:60-63.

20. Bazerbachi F, Dobashi A, Kumar S, et al. Efficacy and safety of combined endoscopic cyanoacrylate injection and balloonoccluded retrograde transvenous occlusion (BRTOcc) of gastrorenal shunts in patients with bleeding gastric fundal varices. Gastroenterol Rep (Oxf) 2020 Dec 03 [Online ahead of print]. doi: 10.1093/gastro/goaa082

21. Li DK, Haffar S, Horibe M, et al. Verrucous esophageal carcinoma is a unique indolent subtype of squamous cell carcinoma: a systematic review and individual patient regression analysis. J Gastroenterol 2021;56:12-24.

22. Jawoosh M, Haffar S, Deepak P, et al. Volvulus of the ileal pouchanal anastomosis: a meta-narrative systematic review of frequency, diagnosis, and treatment outcomes. Gastroenterol Rep (Oxf) 2019;7:403-410.

23. Haffar S, Kaur RJ, Garg SK, et al. Acute pancreatitis associated with intravenous administration of propofol: evaluation of causality in a systematic review of the literature. Gastroenterol Rep (Oxf) 2019;7:13-23.

24. Bazerbachi F, Leise MD, Watt KD, Murad MH, Prokop LJ, Haffar S. Systematic review of mixed cryoglobulinemia associated with hepatitis E virus infection: association or causation? Gastroenterol Rep (Oxf) 2017;5:178-184.

25. Siddappa PK, Hawa F, Prokop LJ, et al. Endoscopic pancreatic duct stenting for pain palliation in selected pancreas cancer patients: a systematic review and meta-analysis. Gastroenterol Rep (Oxf) 2021;9:105-114.

26. Arantes VN, Sanna MDGP, Alves JS, Milhomem-Cardoso DM, Maluf-Filho F. Is endoscopic submucosal dissection still limited to few tertiary centers in the west? Results from a national survey in Brazil. Arq Gastroenterol 2020;57:477-483.

27. Shiga H, Ohba R, Matsuhashi T, et al. Feasibility of colorectal endoscopic submucosal dissection (ESD) carried out by endoscopists with no or little experience in gastric ESD. Dig Endosc 2017;29(Suppl 2):58-65.

28. Pioche M, Rivory J, Nishizawa T, et al. Randomized comparative evaluation of endoscopic submucosal dissection self-learning software in France and Japan. Endoscopy 2016;48:1076-1083.

29. Tanaka S, Oka S, Kaneko I, et al. Endoscopic submucosal dissection for colorectal neoplasia: possibility of standardization. Gastrointest Endosc 2007;66:100-107.

30. Zhang X, Ly EK, Nithyanand S, et al. Learning curve for endoscopic submucosal dissection with an untutored, prevalencebased approach in the United States. Clin Gastroenterol Hepatol 2020;18:580-588.

31. Berr F, Wagner A, Kiesslich T, Friesenbichler P, Neureiter D. Untutored learning curve to establish endoscopic submucosal dissection on competence level. Digestion 2014;89:184-193.

32. Rönnow CF, Uedo N, Toth E, Thorlacius H. Endoscopic submucosal dissection of 301 large colorectal neoplasias: outcome and learning curve from a specialized center in Europe. Endosc Int Open 2018;6:E1340-E1348. 